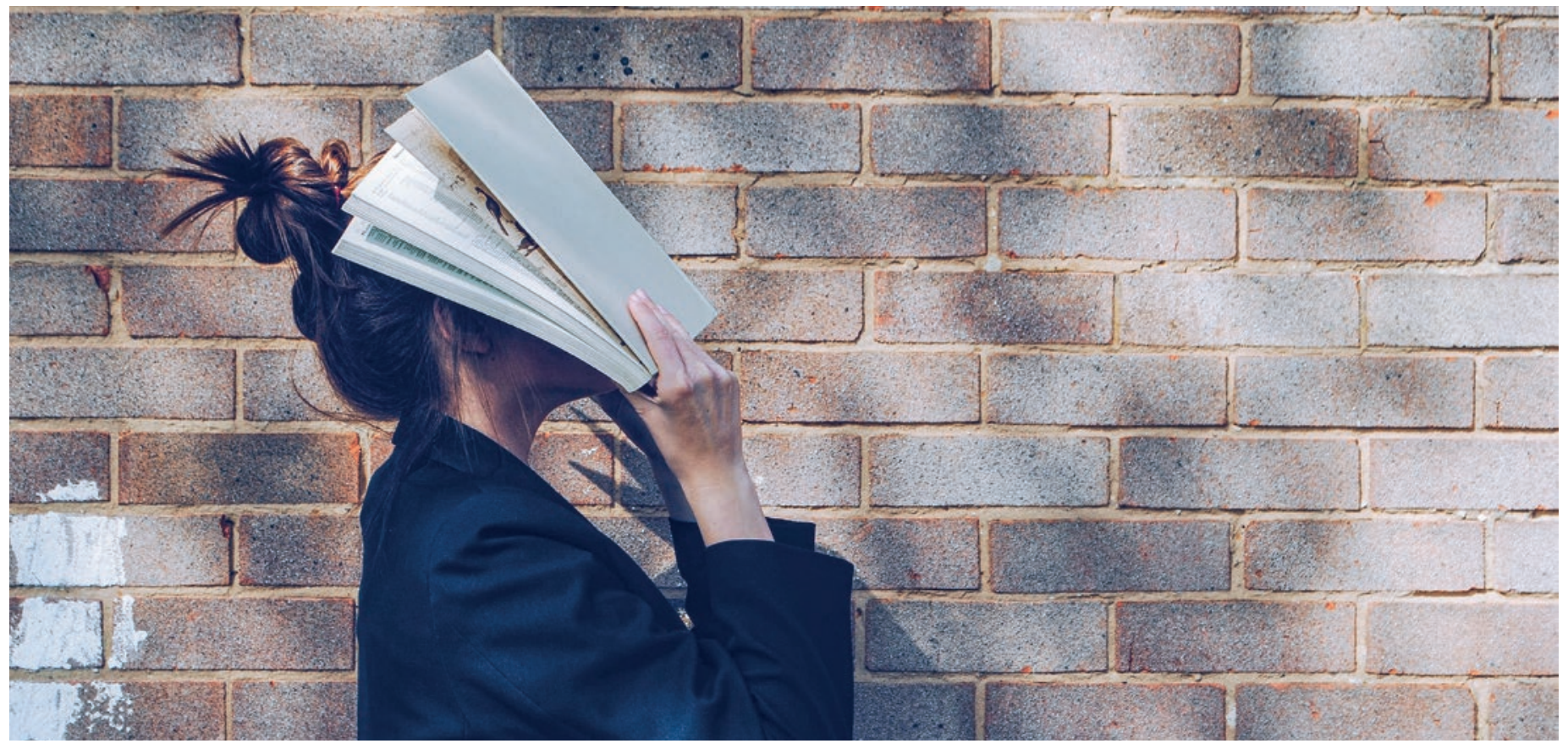

\title{
Wenn alles zu viel wird
}

\section{Lea Luchsinger}

Medizinstudentin, Universität Zürich (St. Galler Track)

Das Medizinstudium ist lang und intensiv, das ist bekannt. Doch wie ist es eigentlich, wenn man mittendrin steckt? Eine Medizinstudentin berichtet darüber, wie sie das zweite Semester erlebt hat und wie es sich anfühlt, alles zu geben und am Ende doch zu scheitern.

Frühlingssemester 2021 - So schnell wie das Semester begonnen hatte, hatte es auch geendet. Wie im Flug war die Zeit vergangen. Doch es war kein leichter Start. Wir begannen mit einem Haufen weit zurückliegender Vorlesungen aus dem Herbstsemester, meinem ersten Semester als Medizinstudentin, die erst im Frühlingssemester geprüft wurden. Das Semester war enorm chaotisch. Wir hatten sehr viele verschiedene Dozierende, die immer wieder ähnliche, aber nicht gleiche Themen behandelten.

\section{Von Anfang an alles geben}

Kein roter Faden, kein Überblick. Praktika, Mantelstudium, Online- und Präsenzunterricht. Schon ganz am Anfang das Gefühl, dass es unmöglich ist: Wie soll man sich so auf Prüfungen vorbereiten? Keine Übungen, keine Lernziele, tausende Folien. Bereits vom Semesteranfang an das Gefühl, in der Lernphase zu sein. Eine immer präsente Panik, es nicht zu schaffen, falsch zu lernen, die Prüfungen nicht zu bestehen. Angst vor dem grossen Scheitern. Die Angst verdrängen und von Anfang an alles geben.

\section{Der Lernstoff wächst und wächst}

Eigene Zusammenfassungen schreiben. Das Gefühl, alles bis aufs Detail verstehen zu müssen. Recherchieren, Bücher lesen und noch einmal zusammenfassen. Aus der Angst, etwas Wichtiges zu verpassen, hatte ich mir auch noch Lernkarten gekauft. Ein riesiger Stapel, hunderte Karten zur Theorie und Aufgaben. Aus den Zusammenfassungen auf die Lernkarten übertragen. Langsam am unüberblickbaren Haufen Lernstoff verzweifeln, mir einreden, dass es gut kommt, dass ich einfach alles geben muss, mehr lernen, länger und intensiver lernen. Wie viel Freizeit kann ich mir trotzdem noch leisten? Ab und zu eine Wanderung, joggen und auch noch selten in der Pfadi auftauchen. 
Das Chaos wurde trotzdem immer grösser. Zu weit habe ich mich von den Vorlesungen entfernt. Ich habe mich immer verbissener aufs Verstehen konzentriert, wollte alles wissen und nicht einfach die Folien auswendig lernen, auch wenn mir eigentlich schon da bewusst war, dass die Folien, der Text auf den Folien, schlussendlich den Prüfungsstoff darstellen.

\section{Immer noch mehr geben}

Immer mehr vom Stress getrieben. Keine Müdigkeit, keine Erschöpfung, keine Grenzen mehr spüren. Immer dieser Gedanke, einfach alles zu geben. Aber im-

\section{Bereits vom Semesteranfang an das Gefühl,} in der Lernphase zu sein. Eine immer präsente Panik, es nicht zu schaffen, falsch zu lernen.

mer das Gefühl, dass es nicht reicht. Gegen Ende der Lernphase lernte ich zwölf bis vierzehn Stunden täglich. Ich konnte nicht mehr wirklich schlafen und war trotzdem noch nicht zufrieden, fühlte mich, als würde ich immer noch nicht genug lernen, nicht alles geben. Mein grosses Interesse, das Recherchieren und Nachlesen brachten mich an den Punkt, an dem ich verstand, wie die Stoffwechselkreisläufe funktionierten, verstand, wie die Zellen aufgebaut sind, wie vom Spermium und der Eizelle ein Fötus entsteht. Jedoch wusste ich nicht, was genau in welchen Worten auf den Folien stand, und das wurde mir schlussendlich auch zum Verhängnis.

\section{Das grosse Scheitern}

Bewusst hatte ich mich entschieden, nicht Folien auswendig zu lernen, sondern zu verstehen. Dies erschien mir sinnvoller, denn nur mit Verständnis kann ich später verknüpfen. Zu wissen, wie was auf der Folie steht, scheint mir nicht viel zu bringen. Schon am Montagmorgen bei der ersten Prüfung wurde mir bewusst, dass ich falsch gelernt hatte.

Es wurde nur sehr wenig Verständnis geprüft. Ich hatte das Gefühl, dass niemand fragte, wieso irgendetwas so ist oder wie etwas funktioniert. Gefühlt waren es nur Fragen danach, ob auf den Folien das oder dies stand, ob es so oder so formuliert wurde, nicht was eine Aussage war oder was sie bedeutete, sondern wer eine Aussage machte. Wie konnte ich mich so auf das Falsche konzentrieren? Wie kann es sein, dass ich so viel gelernt hatte und eigentlich auch verstanden hatte und nun dasitze und nichts beantworten kann? Wie kann das sein? Wie?

Drei Wochen später die Bestätigung: Es hat tatsächlich nicht gereicht. Ich habe die Prüfung nicht bestanden und das auch noch sehr deutlich. 108 Punkte habe ich erreicht, 124 hätte ich gebraucht, die Note ist eine Drei. Im Winter eine Fünf, jetzt eine Drei. Ich habe es nicht geschafft, falsch gelernt, die Prüfung nicht bestanden, mein erstes grosses Scheitern.

\section{Endlich Zeit haben}

Und was jetzt? Für mich war von Anfang an klar, dass ich nicht bereits im Sommer an die Nachholprüfungen gehen werde. Dass ich mir ein Jahr Zeit lassen werde für den zweiten und letzten Versuch. Da ich nach der Matur ohne Zwischenjahr direkt an die Uni gegangen war, schien dies die perfekte Gelegenheit zu sein, dieses nun zumindest zu einem Teil nachzuholen.

Mir wurde, nachdem der erste Schock verarbeitet und die Selbstzweifel beseitigt waren, bewusst, dass es keine Tragödie ist, die Prüfung nicht bestanden zu haben, sondern eine Chance. Eine Chance, endlich einmal Zeit zu haben und nichts zu müssen. Aber was mache ich nun mit dieser plötzlich gewonnenen Freiheit? Interrail, Jakobsweg, Surfen in Portugal oder Hawaii, die Welt sehen, neue Menschen und Kulturen kennen-

Zum jetzigen Zeitpunkt weiss ich noch nicht, was ich genau wann machen werde und muss es auch noch nicht wissen.

lernen, Englisch lernen und sicher auch arbeiten.

Zum jetzigen Zeitpunkt weiss ich noch nicht, was ich genau wann machen werde, und muss es auch noch nicht wissen. Ich weiss jedoch, wie ich mich auf die Prüfungen nächstes Jahr vorbereiten muss, weiss nun, wie und was gefragt wird, werde die Vorlesungen noch einmal besuchen. Ich bin auch schon in Kontakt mit anderen, die wie ich repetieren werden. Ich bin nicht allein und ich werde das hinkriegen. Und falls nicht, werde ich bis dann einen soliden Plan B haben. Und ein Jahr voller wertvoller Lebenserfahrung.

Dieser Bericht entstand im Sommer 2021 im Rahmen eines Mentoringprogramms der Universität St. Gallen und der HSG Alumni, das Studierenden für die Dauer von zwei Jahren den Austausch mit einer Mentorin bzw. einem Mentor ermöglicht.

\section{Bildnachweis}

Siora Photography | Dreamstime.com 